\title{
Electrocatalytic Effect on the Photon-to-Current Conversion Efficiency of Gold-Nanoparticle-Loaded Titanium(IV) Oxide Plasmonic Electrode for Water Oxidation
}

\author{
Tatsuhiro Onishi, ${ }^{[a]}$ Miwako Teranishi, ${ }^{[b]}$ Shin-ichi Naya, ${ }^{[b]}$ \\ Musashi Fujishima, and Hiroaki Tada ${ }^{a, b *}$ \\ [a] Graduate School of Science and Engineering, Kindai University, 3-4-1, Kowakae, \\ Higashi-Osaka, Osaka 577-8502, Japan \\ ${ }^{[b]}$ Environmental Research Laboratory, Kindai University, 3-4-1, Kowakae, Higashi-Osaka, \\ Osaka 577-8502, Japan. \\ [c] Department of Applied Chemistry, Faculty of Science and Engineering, Kindai University, 3-4-1, \\ Kowakae, Higashi-Osaka, Osaka 577-8502, Japan \\ * To whom correspondence should be addressed: TEL: +81-6-6721-2332, FAX: +81-6-6727-2024,
}

E-mail: h-tada@apch.kindai.ac.jp. 

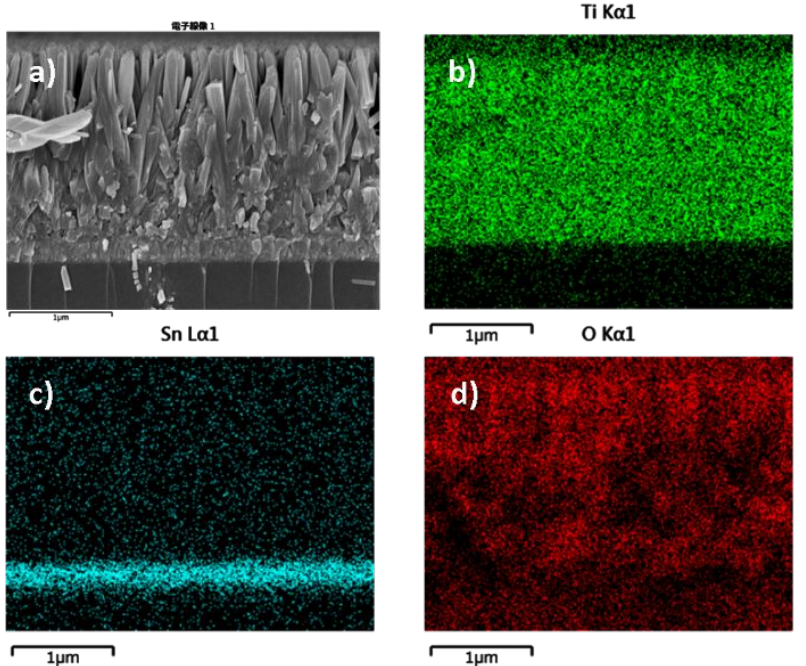

Figure S1. SEM-EDX elemental mapping for a sample prepared by the hydrothermal method.

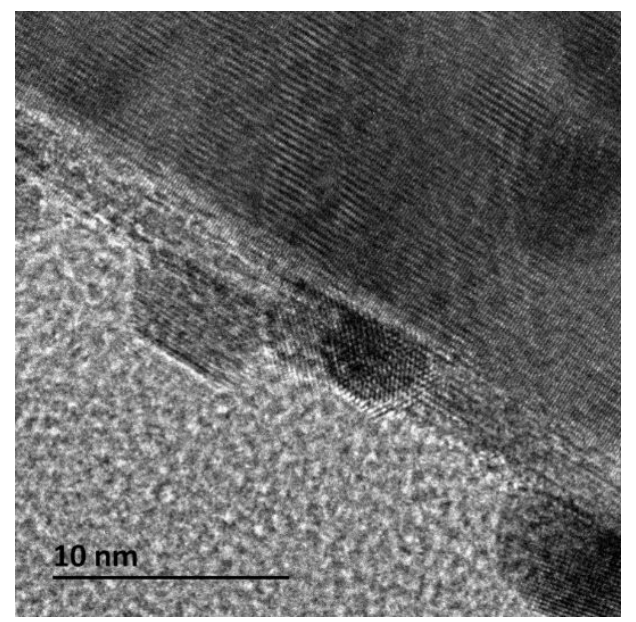

Figure S2. HR-TEM image of Au NPs on rutile $\mathrm{TiO}_{2}$-NWA. 

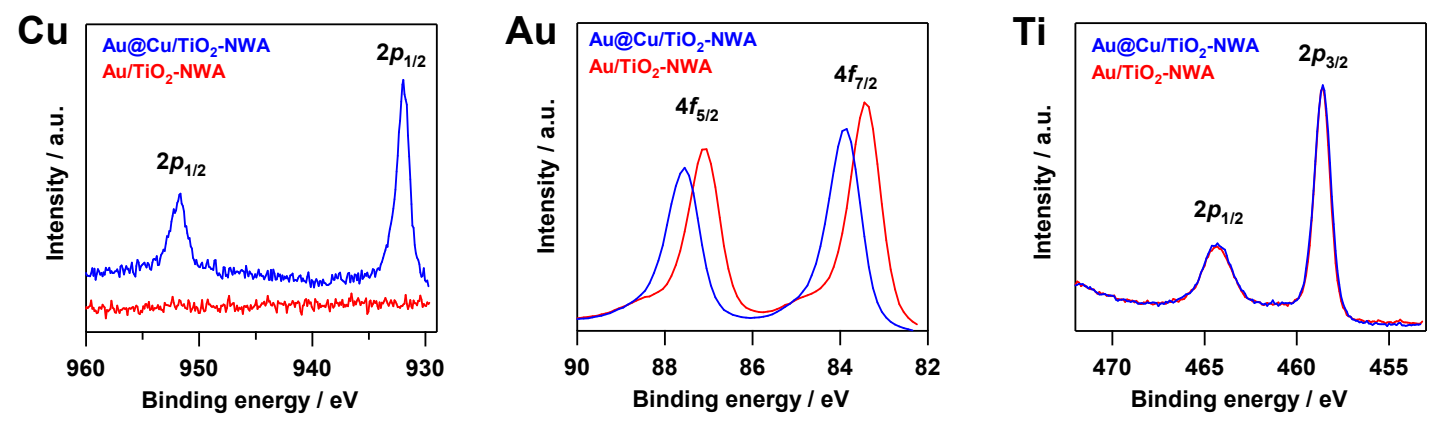

Figure S3. XPS spectra of of $\mathrm{Au} @ \mathrm{Cu} / \mathrm{TiO}_{2}-\mathrm{NWA}$.

\section{Cu shell thickness estimation}

Au mean size $d_{\mathrm{Au}}=5.8 \mathrm{~nm}$

Dispersion (the ratio of surface atom to total atom in nanopartice) $=0.155$

Au loading amount per apparent unit surface area $=63.2 \mathrm{nmol} \mathrm{cm}-2$

$\mathrm{Cu}$ loading amount per apparent unit surface area $=6.85 \mathrm{nmol} \mathrm{cm}{ }^{-2}$

$\mathrm{Cu}$ shell number $=(\mathrm{Cu}$ atom number $) /(\mathrm{Au}$ surface atom number $)$

$=(6.85 \mathrm{nmol} \mathrm{cm}-2 \times$ Avogadro constant $) /$

$\left(63.2 \mathrm{nmol} \mathrm{cm} \mathrm{cm}^{-2} \times\right.$ Avogadro constant $\times$ dispersion)

$$
=0.70
$$



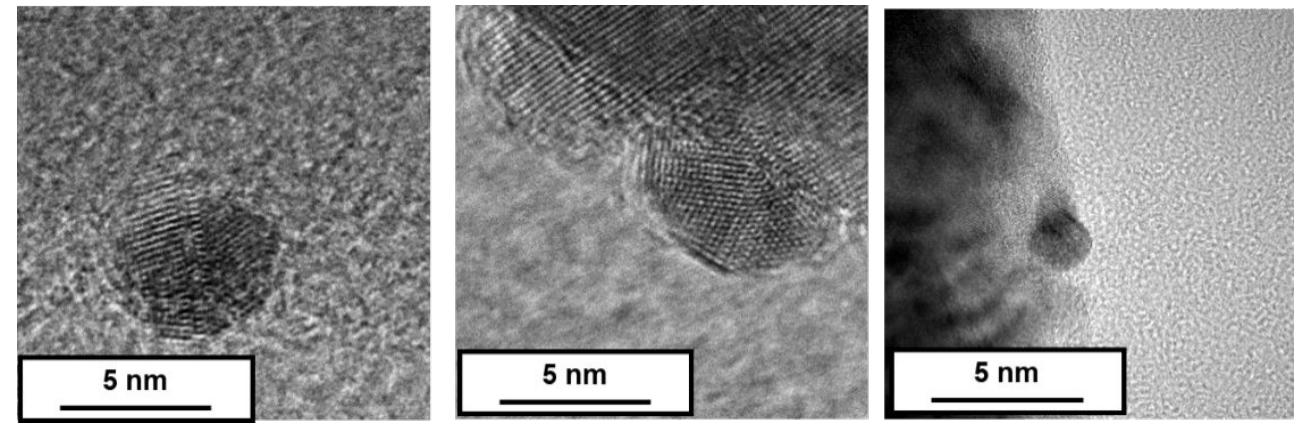

Figure S4. HR-TEM images of $\mathrm{Au} @ \mathrm{Cu} / \mathrm{TiO}_{2}-\mathrm{NWA}$.

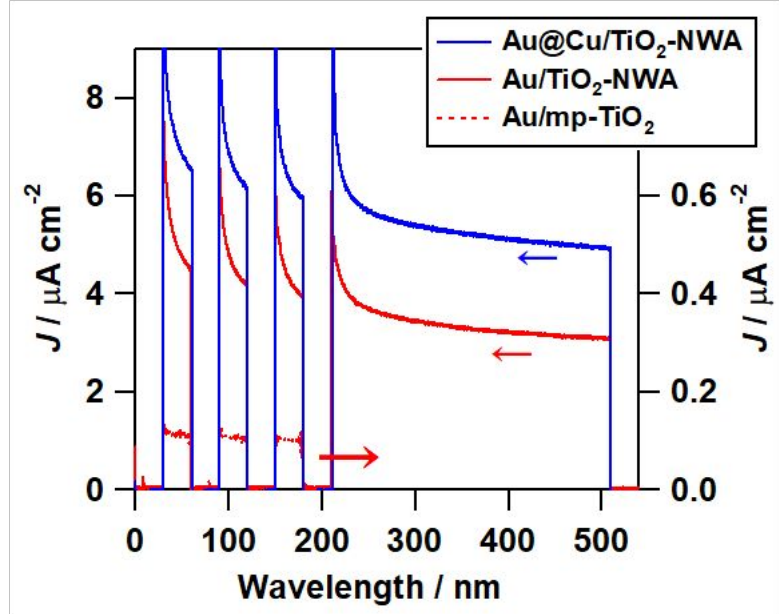

Figure S5. Photochronoamperograms for $\mathrm{Au} / \mathrm{mp}-\mathrm{TiO}_{2} / \mathrm{FTO}, \mathrm{Au} / \mathrm{TiO}_{2}-\mathrm{NWA}$, and $\mathrm{Au} @ \mathrm{Cu} / \mathrm{TiO}_{2}-\mathrm{NWA}$ electrodes at the rest potential in the dark under visible-light irradiation $(\lambda>430 \mathrm{~nm}$, light intensity $=100$

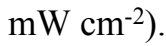




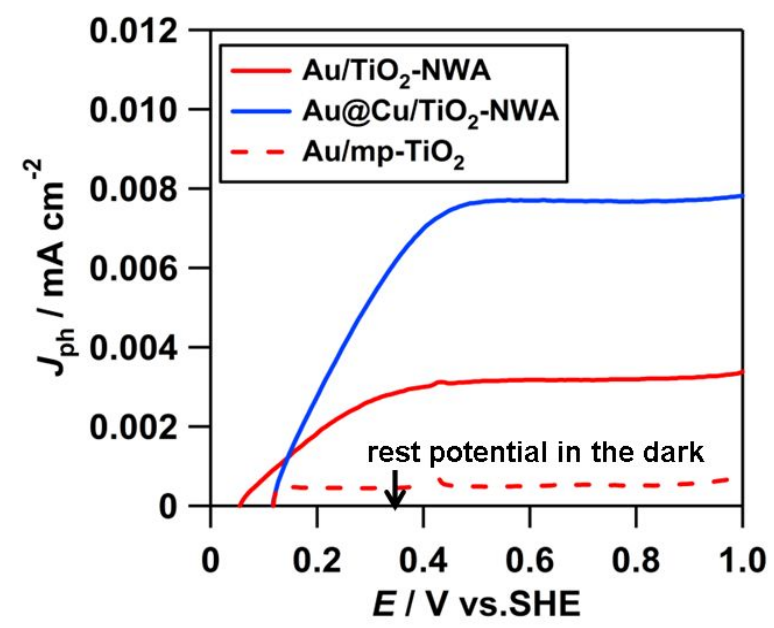

Figure S6. Current-potential curves for $\mathrm{Au} / \mathrm{mp}-\mathrm{TiO}_{2} / \mathrm{FTO}, \mathrm{Au} / \mathrm{TiO}_{2}-\mathrm{NWA}$, and $\mathrm{Au} @ \mathrm{Cu} / \mathrm{TiO} \mathrm{O}_{2}-\mathrm{NWA}$ electrodes at the rest potential in the dark under visible-light irradiation $(\lambda>430 \mathrm{~nm}$, light intensity $=100 \mathrm{~mW}$ $\left.\mathrm{cm}^{-2}\right)$. 